\title{
Multi-Battle Domain-Perspective in Military Medical Simulation Trauma Training
}

Amber S Linde ${ }^{*}$, Lance J McGinnis and David M Thompson

Medical Simulation and Information Sciences, Frederick, Maryland, USA

"Corresponding author: Amber S. Linde, Senior Scientist at Joint Program Committee-1 (JPC-1), Medical Simulation and Information Sciences, Frederick, Maryland, USA, Tel: + 301-619-4051; E-mail: amber.s.linde.civ@mail.mil

Rec date: August 11, 2017; Acc date: August 28, 2017; Pub date: August 30, 2017

Copyright: (C) 2017 Linde AS, et al. This is an open-access article distributed under the terms of the creative commons attribution license, which permits unrestricted use, distribution, and reproduction in any medium, provided the original author and source are credited.

\author{
Abstract \\ On July 24th 2017, the Association of the United States Army (AUSA) held a panel discussion to discuss the \\ United States Army-Marine Corps White Paper entitled, "Multi-Domain Battle (MDB): Combined Arms for the 21st \\ century.
}

Keywords: Multi-Battle; Military medical simulation; Trauma training; Injury; Educational gaming; Emerging military

\section{Introduction}

The AUSA panel emphasized that prolonged field care (PFC) at the point of injury and the point of care will become the norm. It is predicted that medical evacuation routes will be limited due to force dispersion, leading to delays or absence in medical evacuation of the casualty. It is expected that without a planning mechanism in place regarding new Theatre pre-hospital trauma care tactics, a decreased personnel survivability and higher casualty numbers will result $[1,2]$. In short, "U.S. forces are not organized, trained, equipped, and postured to properly contest emerging and potential threats" [2].

Currently the USA does not have federal institutes or private foundations specifically for trauma patients at any role of care. This is concerning, given the increased risk of future wars involving predicting far more casualties [3]. The "golden hour" is defined as "delivering advanced resuscitative capability to the injured person within one hour, regardless of location or echelon of care" (Col Todd E. Rasmussen, USAMRMC CCCRP). Future combat scenarios will increase the use of small troop groups working in remote sites, large combat operations in the Pacific theater, and large combat forces operating in mega-cities; all these situations will increase the chances of high numbers of civilian and military casualties [3]. The future MDB is further challenged regarding the probable absence of MEDEVAC services and current availabilities of medical care close to the battlefield. New solutions to these concerns and challenges need to allow medical staff in a pre-hospital Theatre setting to support a large number of casualties that will not have the luxury of being moved quickly to a stable/safe medical facility [4].

\section{Military Medical Simulation}

The Medical Simulation and Information Sciences Research Program (MSISRP) was stood up in 2010 and is part of the USA Army Medical Research and Materiel Command's (USAMRMC) research area directorate. The MSISRP is tasked with planning, coordinating, and overseeing a tri-service science and technology program to improve strategic planning and process development by improving military medical training through medical simulation, educational gaming, and objective training metrics and improving health information sciences through increased interoperability and better health information technology applications [5].

The mission of the MSISRP is to coordinate emerging military medical simulation and health information research across all stakeholder communities and transfer research solutions and knowledge to meet Military Health System (MHS) goals. Based on the concepts presented at AUSA and items documented in the white paper, it is clear that the way health simulation and health information technology is currently delivered will be evolving rapidly, in order to support service members on a future battlefield.

In order to successfully deliver MDB solutions, the MSISRP needs to understand the potential operational environments it is meant to address. The final percentage of combat-related deaths (2000-2015) revealed that $76 \%$ of combat deaths occurred during point of care while the patient was in the theater pre-hospital setting [6]. It is urgent for the Department of Defense (DoD) to address and discover new knowledge/materiel and training solutions for the future battlefield scenarios such as Prolonged Field Care (PFC), long-distance air- landor sea-based medical evacuation [7].

As more details are provided, MSISRP will be developing, testing and evaluating, and applying current and upcoming research projects that can directly influence how we provide support to battlefield medicine ensuring MDB's success in theatre healthcare. Early implementation of the MDB will be focus on "concept development, war -gaming, experimentation and capability development" [2]. The MDB systems are required to provide flexible and resilient platforms that can handle threats from a multi-plane battlefield [4]. PFC simulation training needs to incorporate all battlefield domains (whether providing self or buddy aid, or PFC methods) in air, land, sea, space, cyberspace, electromagnetic spectrum (EMS), military information support operations, physical attack, special technical operations, information assurance, computer network operations and civil-military operations [2].

To meet future challenges facing America's Warfighters, the Defense Health Agency (DHA) developed a medical simulation enterprise consisting of six integrated and federated programs. The very first of these six programs to be launched is the Joint Evacuation and Transport Simulation (JETS) program. This program includes research 
Page 2 of 2

efforts focused on bridging validated gaps across the MHS as they are identified in multiple DoD requirements documents with respect to global/joint patient movement. As the first DoD-wide program, JETS set a historical and ground-breaking milestone, becoming the first joint service medical simulation program in the history of the DoD (Figure $1)$.

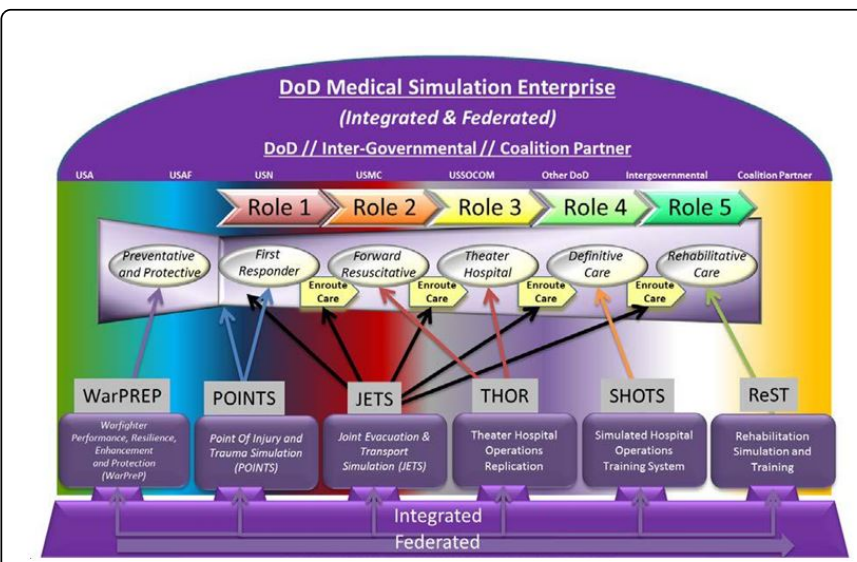

Figure 1: DoD Medical Simulation Enterprise in 2017. Medical simulation and information research sciences portfolio. Internal brief.

The JETS program will link the operational needs of component and geographic combatant commands. This will have standardized DoD patient movement training platform which will directly support component/service training missions resulting in the sustainment of clinical standards for patient management. The end-state is to ensure patients receive the most effective care throughout the patient movement process in the DoD chain of evacuation. The JETS platform is expected to decrease the DoD's training costs and technology fielding timelines without affecting the Components' training authorities. JETS is expected to provide augmentation of training capabilities already in place, while adding sustained capability into the future.
The JETS system supports skills and higher-level learning for individual, team and unit training of patient movement tasks covering the complete chain of patient evacuation, throughout the continuum of care. It delivers a global point of demand training capability, linking training centers and warfighters around the globe, 24 hours a day, 7 days a week, and 365 days a year. The JETS system gives users the capability to conduct integrated, live, virtual, constructive and gaming methods of training within a medical synthetic training environment that is delivered through DoD training portals.

\section{Conclusion}

The MISISRP seeks to meet the unpredictable challenges of the multi-domain battle with innovative, integrated and federated medical simulation technologies to train and sustain the skills needed that will support the safe and effective movement of patients from the battlefield to the next level of care and throughout the evacuation continuum of care.

\section{References}

1. AUSA 2017-Future modernization to enable multi domain battle - Panel Discussion. (2017). AUSA Institute of Land Warfare Global Force Symposium and Exposition.

2. United States Army-Marine Corps White Paper (2017) Multi-domain battle: Combined Arms for the 21st Century.

3. Rasmussen CE, Baer DG, Doll BA, Caravalho J (2015) In the golden hour: Combat casualty care research drives innovation to improve survivability and reimagine future combat care. Army AL and $\mathrm{T}$ Magazine 80-85.

4. Rasmussen TE, Baer DG, Remick KN, Ludwig GV (2017) Combat casualty care research for the multidomain battlefield. J Trauma Acute Care Surg 83: S1-S3.

5. Medical Simulation and Information Research Sciences Portfolio (2015).

6. Perkins DG (2017) Multi-domain battle driving change to win in the future. Military Review 6-12.

7. Rasmussen TE, Baer DG, Cap AP, Lein BC (2015) Ahead of the curve: Sustained innovation for future combat casualty care. J Trauma Acute Care Surg 79: S61- ${ }^{\mathrm{S}} 64$. 